\title{
GALEX Ultraviolet Observations of Globular Clusters in Nearby Galaxies
}

\section{Soo-Chang Rey ${ }^{1}$, Sangmo T. Sohn ${ }^{2}$, R. Michael Rich ${ }^{3}$, Suk-Jin Yoon $^{2}$, Chul Chung ${ }^{2}$, Sukyoung K. Yi ${ }^{2}$, and Young-Wook Lee ${ }^{2}$}

${ }^{1}$ Department of Astronomy and Space Science, Chungnam National University, Daejeon 305-764, Korea

email: screy@cnu.ac.kr

${ }^{2}$ Center for Space Astrophysics, Yonsei University, Seoul 120-749, Korea

${ }^{3}$ Department of Physics and Astronomy, University of California at Los Angeles, Box 951547, Knudsen Hall, Los Angeles, CA 90095

The hot He-burning horizontal-branch (HB) stars and their progeny are most likely dominant ultraviolet (UV) sources in the old stellar population systems such as globular clusters (GCs). Integrated FUV flux can be an age indicator of GCs and allow us to investigate age distributions of GCs within a given galaxy or between galaxies. The unprecedented set of UV photometry for M31 by Galaxy Evolution Explorer (GALEX), coupled with most recent detailed population models enable to study detailed global UV properties of M31 GCs.

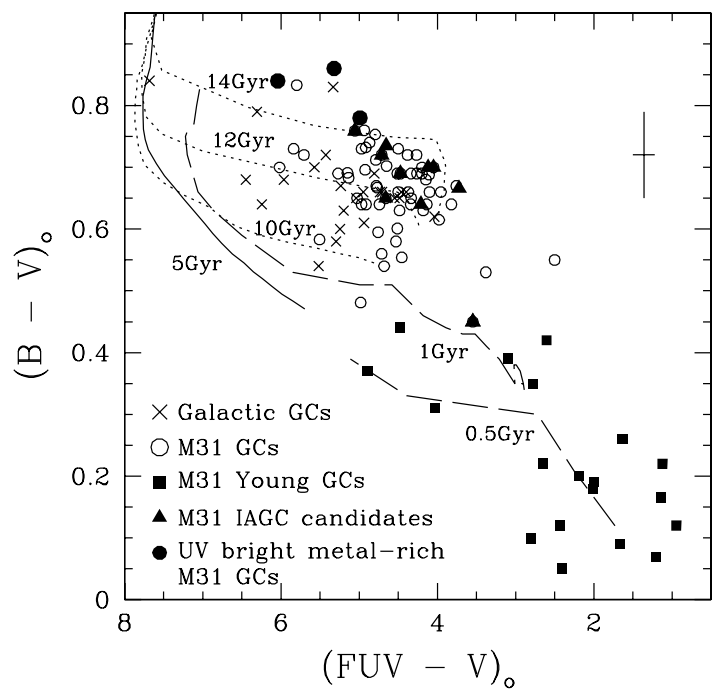

Reference

Rey, S.-C. et al. 2006, ApJS, in print
Figure 1. $(B-V)_{0}$ vs. $(F U V-V)_{0}$ in M31 and Galactic GCs. Our model isochrones for different ages are also superposed. Three metal-rich $([\mathrm{Fe} / \mathrm{H}]>-1)$ M31 GCs (filled circles) show significant FUV flux comparable to those of NGC 6388 and NGC 6441, peculiar metal-rich Galactic GCs with anomalous hot horizontal-branch (HB) stars. Among intermediate-age GC (IAGC, 4-7 Gyr) candidates proposed by recent spectroscopic observations, many GCs are detected in GALEX observations (filled triangles). We suggest that some of spectroscopically identified IAGCs may not be truly intermediate in age, but rather older GCs with developed HB stars. We confirm that there are significant fraction of young GCs (filled boxes) in M31 (see Rey et al. (2006) for the details). 